\title{
Diagnóstico, diseño y evaluación económica del sistema de riego por aspersión del bosque el Olivar
}

\author{
Diagnosis, design and economical assessment of sprinkler irrigation system of bosque el Olivar
}

\author{
David Ascencios T. ${ }^{1}$; Peña A. ${ }^{2}$
}

\begin{abstract}
Resumen
En el año 1560 se trajeron a Lima los primeros almácigos de olivos que dieron origen al "Bosque del Olivar" y, en 1959, el Olivar fue declarado Monumento Nacional por el Instituto Nacional de Cultura (INC); en este contexto, toma especial importancia la preservación de los olivos del lugar puesto que el inadecuado manejo agronómico y del recurso hídrico actual podrían poner en serio peligro este patrimonio. El presente estudio evalúa las alternativas, desde el punto de visto técnico y económico, del cambio del sistema de riego de gravedad a aspersión (difusores) en el Bosque del Olivar. Para el estudio se realizó un diagnóstico del riego actual del área, así como de la fuente de agua, la energía, el clima, el suelo y los cultivos, para luego formular el proyecto desarrollando el balance hídrico y los diseños agronómico e hidráulico, necesarios en la formulación de la propuesta técnica para implementar el riego por aspersión. Esta propuesta considera almacenamiento (cisternas), estación de bombeo, cabezal de control, red matriz y distribución, zonas de riego, emisores y automatización. El cambio del sistema de riego permitirá mantener al cultivo de olivo en mejores condiciones agronómicas y realizar un uso eficiente del recurso hídrico. La evaluación económica realizada consiste en la estimación de los costos actuales y la respectiva proyección con el proyecto y el análisis de beneficios, determinándose un Valor Actual Neto (VAN) de S/. 1.748.633,04, con una tasa de 11 \%, una TIR de $27 \%$ y un ratio beneficio-costo (B/C) de S/. 1,51.
\end{abstract}

Palabras clave: diagnóstico; diseño; evaluación; aspersión.

\begin{abstract}
In 1560, the first olive seedbeds were brought to Lima, which gave rise to the "Bosque el Olivar", and in 1950, the "Olivar" was declared National Monument by the National Institute of Culture (INC); in this context, the preservation of olive trees of the park takes special importance, highlighting the esthetic character and taking into consideration the inappropriate agronomic and water resources management, which would seriously endanger this heritage. This study assessment the alternatives, from the technical and economic point of view, of the change of the irrigation system of gravity to a sprinkler irrigation system (diffusers) at the "Bosque del Olivar". A diagnosis of the current irrigation at the "Olivar" was held, considering the source of water, energy, climate, soil and crops; for then formulate the project developing the water balance, agronomic and hydraulic design, needed in the formulation of the technical proposal to implement sprinklers irrigation system, which considers the storage (cisterns), pumping station, head control, man and sub main pipe network, irrigation zones, emitter and automation areas. The change of the irrigation system will allow keeping olive growing in better agronomic conditions and get a better efficient use of the water resources. The economic assessment consists of the estimate of the current and projected costs with the project and the analysis of benefits, both from a qualitative and quantitative point of view, that determine a NPV of S/. 1.748.633,04, with a rate of $11 \%$, an IRR of $27 \%$ and a benefit-cost ratio (BCR) of S/. 1,51.
\end{abstract}

Keywords: diagnosis; design; evaluation; sprinkler.

\section{Introducción}

Nicolás de Rivera, fundador de la Ciudad de los Reyes en 1560, siendo Alcalde, trajo los primeros almácigos de olivos que dieron origen al "Bosque del Olivar". En el año 1931, con la creación del distrito de San Isidro, se delimitó el tamaño con el que actualmente se conoce al bosque. En
1959, El Olivar fue declarado Monumento Nacional por el Instituto Nacional de Cultura (INC). En este contexto, cobra especial importancia la preservación de los olivos, predominando el carácter estético y patrimonial. En la actualidad, presenta enfermedades fitosanitarias producto del inadecuado manejo agronómico y del recurso

1. Universidad Nacional Agraria La Molina, Lima (Perú). Email: dascencios@lamolina.edu.pe

2. Universidad Nacional Agraria La Molina, Lima (Perú). Email: marcelo_peal@yahoo.es 
hídrico que ponen en serio peligro este patrimonio. La responsabilidad de conservación de un patrimonio histórico y el uso eficiente del recurso hídrico, motivan el presente estudio.

El mantenimiento del bosque requiere de una especial dedicación, sin embargo se afrontan una serie de inconvenientes para conservar el área en óptimas condiciones:

- El sistema actual de riego por inundación es incompatible con los requerimientos hídricos distintos, en cantidades y frecuencias, de las dos especies que conviven en la zona, el olivo (Olea europea) y el césped.

- El abastecimiento de agua es deficiente en cantidad, calidad y oportunidad.

- El empleo de técnicas adecuadas para el uso eficiente del agua está ausente.

- Las áreas de riego están desniveladas.

- Las aguas que se usan provienen del río Surco y son de muy baja calidad desde el punto de vista de la inocuidad.

- La carencia de personal especializado en manejo agronómico es notoria.

Las deficiencias antes mencionadas acarrean como consecuencias:

- Desperdicio del agua de riego, cuya disponibilidad será crítica a muy corto plazo.

- Alta proliferación de insectos y roedores.

- Inundación de los árboles de olivo, en períodos muy frecuentes y en momentos que esta especie vegetal no requiere agua, afectando el sistema radicular del olivo.

- Presencia de un alto índice de enfermedades en los árboles de olivo, a consecuencia del sistema de riego empleado.

La necesidad de cambio del sistema de riego del Bosque del Olivar se relaciona con las deficiencias en la administración del riego. Cabe mencionar que es de interés de la comuna de San Isidro la sostenibilidad del bosque en el tiempo, por lo cual el municipio promueve un nuevo sistema de riego a fin de asegurar su preservación.

El objetivo del presente estudio es evaluar las alternativas técnicas y económicas del nuevo sistema de riego, a aspersión (difusores) en vez de gravedad, en el Bosque del Olivar. El área de estudio se circunscribe al Bosque del Olivar, ubicado en el distrito de San Isidro, departamento de Lima. El bosque tiene una extensión de 10,7 ha.

\section{Materiales y métodos}

Se usaron los siguientes materiales: Plano topográfico y planimétrico a detalle, a escala de $1 / 750$, datos climatológicos de la estación Alexander Von Humboldt; correntómetro para los aforos (cantidad); tarifario del arbitrio del distrito de San Isidro; datos demográficos; cultivos; costos de energía; muestras de agua (calidad) y suelo; encuesta aplicada a los vecinos y visitantes del distrito; computadora; software Autocad, Epanet; costos de mano de obra; materiales; equipos y rendimientos.
Se realizó la siguiente secuencia de actividades:

- El análisis se inicia con un diagnóstico del riego actual de la zona, que incluye la infraestructura de riego, las acciones de operación y mantenimiento, características del terreno, climatología, dotación de agua y calidad, considerando el contenido de elementos tóxicos y la concentración de sales.

- Se realiza un balance hídrico actual y con proyecto, se analiza la oferta actual del recurso hídrico, para luego ser comparada con la demanda actual y proyectada. Se determina los parámetros de diseño para los cultivos, a fin de conservar la estética del bosque.

- Se desarrolla el diseño agronómico e hidráulico, metrados, costos y presupuestos necesarios para el desarrollo de la propuesta técnica de riego por aspersión (difusores), considerando la fuente de agua, fuente de energía, suelos, sistemas de almacenamiento (cisternas), red principal y distribución, emisores y automatización.

- La evaluación económica contempla la estimación de los costos actuales del mantenimiento del bosque, el costo de inversión requerido y la estimación de los costos de operación y mantenimiento que el proyecto generaría.

- Se desarrolla el análisis de beneficios, tanto desde el punto de vista cualitativo como cuantitativo; respecto de los beneficios cuantitativos, se estimará el valor presente del bosque, el valor de la producción del olivo y la valorización de la potencialidad de producción de bonos de carbono del bosque.

- El análisis de rentabilidad se desarrollará bajo la metodología del Costo - Beneficio, en la que el flujo de caja del proyecto estará en función de los costos del proyecto y los ingresos cualitativos antes mencionados que potencialmente pueden captarse. Los indicadores de rentabilidad a trabajar serán el Valor Actual Neto (VAN), la Tasa Interna de Retorno (TIR) y el Ratio Beneficio - Costo (B/C).

- Finalmente, se desarrollarán las conclusiones a las que llegará el estudio y las recomendaciones.

\section{Resultados y discusión \\ Infraestructura de riego actual}

El sistema de conducción del sistema de riego en el distrito de San Isidro se compone de una red de canales enterrados, de sección rectangular y/o circular. La red de distribución principal interna del bosque la conforman canales circulares enterrados de PVC y cemento, cuyos diámetros varían entre 8", 10" y 12". Los canales de distribución principales recorren la calle Antero Aspíllaga y el Paseo Padre Constancio Bollar, a lo largo de su recorrido se presentan cajas a distanciamientos variables de 10 y 20 metros, que sirven de puntos para el riego por inundación y/o conexiones a otras cajas de distribución interna.

Operación y mantenimiento de las áreas verdes 
El Bosque el Olivar, originalmente, surgió como un huerto de olivos. Durante el proceso de consolidación urbana, cobró importancia el ornato sobre la productividad agrícola, cubriéndose el huerto de césped y dando origen al imponente bosque de olivos que vemos hoy. Sin embargo, ello generó un conflicto entre las necesidades culturales para el mantenimiento de estas áreas verdes. Actualmente, el césped es podado con un pequeño tractor para las zonas amplias y una desbrozadora para las áreas irregulares y bordes. El corte se realiza cada 20 días en invierno y 15 días en verano. Los olivos son lavados después de la cosecha, con mangueras a presión. Se los fertiliza una vez al año, aplicando pesticidas y abono orgánico en la proyección de la copa del árbol.

El riego del bosque se realiza por inundación, de manera manual, con la misma tradición que en sus orígenes. Este proceso se realiza los días lunes y jueves, a excepción del mes de agosto en que se realiza la limpieza de canales. El sistema de riego artesanal con cierta frecuencia presenta inundaciones y atoros. Por los desniveles existentes en el terreno, se originan grandes charcos durante el riego, teniendo como resultado una baja eficiencia de aplicación y distribución del mismo (ver Fig. 1).

La limitada administración técnica del riego provoca su sobre dimensionamiento, respecto a la cantidad requerida por los olivos e, incluso, en algunos meses del año, en el césped, deteriorando progresivamente el estado fitosanitario del bosque.

Ante la convivencia de dos especies vegetativas de distintas necesidades hídricas y baja eficiencia de riego, siendo la del césped de mayor demanda frente al olivo, el riesgo para la continuidad de los árboles es latente, poniéndose en riesgo el deterioro de un patrimonio cultural y monumental de la nación.

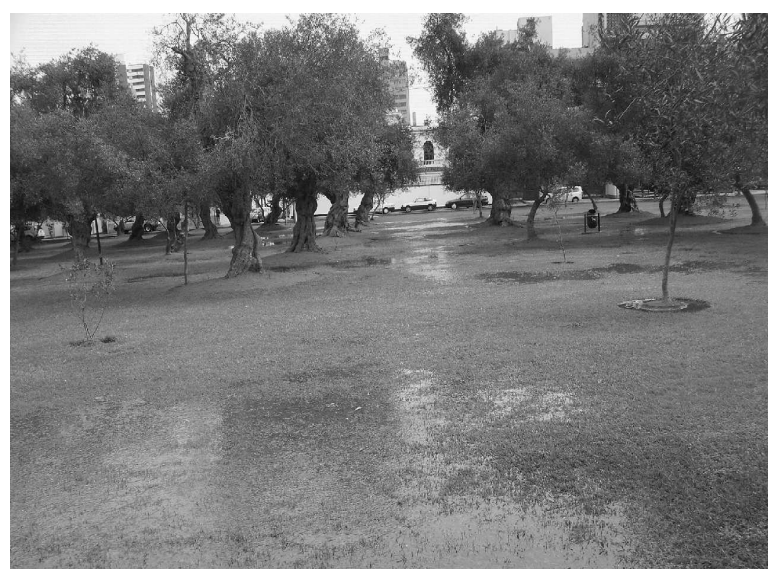

Figura 1. Riego por inundación.

\section{Características del terreno}

El terreno del bosque presenta un relieve no uniforme que dificulta el mantenimiento de las áreas verdes y la administración del riego. El terreno tiene una pendiente promedio de $1,44 \%$.

Desde el punto de vista edafológico, el tipo de suelo del bosque es franco, con una buena aptitud para el riego, presentando los siguientes coeficientes hídricos: Capacidad de campo y punto de marchitez de $32 \%$ y $16 \%$, respectivamente (Vera y Moreno Consultores, 2006).

\section{Clima}

El clima de Lima se caracteriza por sus altos niveles de humedad atmosférica y su escasa precipitación. Lima se ubica en una zona tropical, a 12 grados latitud sur y casi al nivel del mar, presenta una temperatura máxima promedio de $23,2^{\circ} \mathrm{C}$, en febrero, y temperatura mínima de $15,6{ }^{\circ} \mathrm{C}$, en agosto.

La humedad relativa máxima promedio es de $88 \%$ en agosto y la mínima de $78 \%$ en febrero. Los vientos máximos promedio son de $196,8 \mathrm{~km} /$ día, en febrero, y la mínima de $133,7 \mathrm{~km} /$ día, en agosto.

El tanque evaporímetro ha registrado una evaporación máxima promedio de $170,2 \mathrm{~mm} /$ día, en marzo, y $65 \mathrm{~mm} /$ día en junio y julio.

\section{Recursos hídricos}

La fuente de agua proviene del río Rímac, la cual es captada por el canal de derivación denominado río Surco. El agua ingresa al área de estudio con un caudal de 50 1/s, transportado por una tubería circular de PVC de 10 pulgadas.

La Comisión de Regantes del sub-sector de riego del río Surco establece como turnos de riego semanales para San Isidro, los días lunes de 6 am a 6 pm (12 horas) y los jueves de 6 pm a 6 am (12 horas).

\section{Calidad del agua}

Los parámetros microbiológicos obtenidos no se encuentran dentro de los límites permisibles recomendables por la Ley de Recursos Hídricos para el riego de parques y jardines, siendo necesaria una planta de tratamiento de aguas previa al uso de agua para parques y jardines utilizando difusores.

Los resultados obtenidos en el análisis de laboratorio presentan los índices de $\mathrm{CE}$ de $0,71 \mathrm{mS} / \mathrm{cm}$, que se encuentran dentro del rango $0,25-0,75 \mathrm{mS} / \mathrm{cm}$; tales índices corresponden a un agua de salinidad media (C2) que puede usarse siempre y cuando haya un grado moderado de lavado.

\section{Relación de adsorción de sodio (RAS)}

De acuerdo con la información de las aguas del río Surco, la alcalinización media por la relación de adsorción de sodio (RAS) es de 0,88. Valor que de acuerdo a la clasificación de aguas con relación al RAS, se considera agua baja de sodio (S1), la cual puede usarse para el riego en la mayoría de los suelos y tiene poca probabilidad de alcanzar niveles peligrosos de sodio intercambiable.

\section{Índice de saturación de Langelier (ISL)}

Resultados calculados de acuerdo al software de calculadora del Índice de Saturación Langelier de LENNTECH, son pH 7.637 y ISL -0.017. De acuerdo a los valores obtenidos, se puede considerar que el agua es no saturada con respecto a carbonato de calcio $(\mathrm{CaCO} 3)$, de esta forma nos aseguramos que por la calidad de agua no hay tendencia a la formación de incrustaciones. 


\section{Balance hídrico \\ Oferta de agua}

El caudal derivado hacia la toma de captación de la cuadra 28 de la Av. Arequipa, es de aproximadamente 50 $1 / \mathrm{s}$, que equivalen a $4.320 \mathrm{~m}^{3}$ por semana ( 2 turnos, 12 horas /turno), considerando 48 semanas al año, se obtiene un total de $207.360 \mathrm{~m}^{3}$ como promedio anual. Cabe mencionar que la dotación semanal es la misma durante todo el año.

\section{Demanda de agua del olivo}

Para el cálculo de la evapotranspiración de los cultivos, se ha utilizado el método propuesto por la FAO, tomando como base la evaporación del tanque de la estación Alexander Von Humboldt y el coeficiente del tanque y cultivo respectivo. Es importante hacer la acotación que para efectos de cálculo de la demanda hídrica actual, se consideró una eficiencia de riego del $50 \%$ en la cabecera del bosque para riego por gravedad (inundación), en un área de 10,7 ha; al ejecutarse el proyecto, la eficiencia de riego se incrementaría a $85 \%$ en la cabecera del bosque, lo cual reducirá considerablemente las necesidades del recurso hídrico.

\section{Balance hídrico actual}

Al comparar la dotación de agua y su aplicación, bajo las condiciones actuales, se evidencia que, en las 10,7 ha que conforman el Bosque del Olivar, para el caso del césped existe un déficit de agua, especialmente en los meses de verano, generándose un déficit anual de $26.919 \mathrm{~m}^{3}$ (ver
Tabla 1). Bajo el supuesto de que las 10,7 ha del bosque solo están cubiertas por olivos, en función a los cálculos realizados se ha identificado que existe sobre aplicación de agua, de $66.661 \mathrm{~m}^{3}$ acumulados anuales (ver Tabla 2). El balance hídrico sin proyecto permite conocer la actual situación del sistema de riego, determinándose la existencia de una sobre oferta de agua para el Olivar, por el método de riego empleado, el cual produce encharcamientos e inundación en las áreas cercanas a las raíces produciendo pudrición de las mismas.

\section{Balance hídrico proyectado}

El balance de agua proyectado de las 10,7 ha del bosque, para el caso del césped, dado que se incrementará la eficiencia de riego, se estima que se estaría superando el déficit identificado en la situación actual, pasando a una sobre oferta del recurso durante todos los meses, acumulándose $69.549 \mathrm{~m}^{3}$ anuales (ver Tabla 3).

Para el olivo, que es un especie que requiere de poca agua y dado que la eficiencia de riego ha mejorado en un $85 \%$, la sobre oferta de agua sería de $124.673 \mathrm{~m}^{3}$ anuales, ver Tabla 4. En función a los resultados obtenidos, se presenta que el olivo tiene una demanda hídrica mucho menor que la del césped; para efectos de cálculo del volumen de agua a requerir, se utilizará el que demande el césped para implementar el nuevo sistema de riego.

\section{Diseño agronómico}

A continuación, se presentan los cálculos de diseño agronómicos requeridos en el planteamiento del proyecto

Tabla 1. Balance hídrico actual para el césped

\begin{tabular}{|c|c|c|c|c|c|c|c|c|c|c|c|c|c|}
\hline Descripción & $\mathbf{E}$ & $\mathbf{F}$ & $\mathbf{M}$ & $\mathbf{A}$ & M & $\mathbf{J}$ & $\mathbf{J}$ & $\mathbf{A}$ & $\mathbf{S}$ & $\mathbf{O}$ & $\mathbf{N}$ & D & $\begin{array}{c}\mathrm{mm} / \\
\text { anual }\end{array}$ \\
\hline $\begin{array}{l}\text { Oferta } \\
\left(\mathrm{m}^{3} / \mathrm{mes}\right)\end{array}$ & 17280 & 17280 & 17280 & 17280 & 17280 & 17280 & 17280 & 17280 & 17280 & 17280 & 17280 & 17280 & 207360 \\
\hline $\begin{array}{l}\text { Demanda } \\
\left(\mathrm{m}^{2} / \mathrm{mes}\right)\end{array}$ & 26410 & 25399 & 27292 & 25024 & 17482 & 11794 & 11812 & 12121 & 15193 & 20355 & 21371 & 20026 & 234279 \\
\hline $\begin{array}{l}\text { Déficit/ } \\
\text { exceso }\end{array}$ & -9130 & -8119 & 10012 & -7744 & -202 & 5486 & 5468 & 5159 & 2087 & -3075 & -4091 & -2746 & -26919 \\
\hline
\end{tabular}

Tabla 2. Balance hídrico actual para el olivo

\begin{tabular}{|c|c|c|c|c|c|c|c|c|c|c|c|c|c|}
\hline Descripción & $\mathbf{E}$ & $\mathbf{F}$ & M & $\mathbf{A}$ & M & $\mathbf{J}$ & $\mathbf{J}$ & $\mathbf{A}$ & $\mathbf{S}$ & $\mathbf{O}$ & $\mathbf{N}$ & D & $\begin{array}{c}\mathrm{mm} / \\
\text { anual }\end{array}$ \\
\hline $\begin{array}{l}\text { Oferta }\left(\mathrm{m}^{3} /\right. \\
\mathrm{mes})\end{array}$ & 17280 & 17280 & 17280 & 17280 & 17280 & 17280 & 17280 & 17280 & 17280 & 17280 & 17280 & 17280 & 207360 \\
\hline $\begin{array}{l}\text { Demanda } \\
\left(\mathrm{m}^{3} / \mathrm{mes}\right)\end{array}$ & 15846 & 15240 & 16375 & 15015 & 10489 & 7077 & 7087 & 7273 & 9116 & 12212 & 12822 & 12015 & 140567 \\
\hline $\begin{array}{l}\text { Déficit/ } \\
\text { exceso }\end{array}$ & 1434 & 2040 & 905 & 2265 & 6791 & 10203 & 10193 & 10007 & 8164 & 5068 & 4458 & 5265 & 66793 \\
\hline
\end{tabular}

Tabla 3. Balance hídrico proyectado para césped

\begin{tabular}{cccccccccccccc}
\hline Descripción & $\mathbf{E}$ & $\mathbf{F}$ & $\mathbf{M}$ & $\mathbf{A}$ & $\mathbf{M}$ & $\mathbf{J}$ & $\mathbf{J}$ & $\mathbf{A}$ & $\mathbf{S}$ & $\mathbf{O}$ & $\mathbf{N}$ & $\mathbf{D}$ & $\begin{array}{c}\mathbf{m m} / \\
\text { anual }\end{array}$ \\
\hline $\begin{array}{c}\text { Oferta ( m3/ } \\
\text { mes) }\end{array}$ & 17280 & 17280 & 17280 & 17280 & 17280 & 17280 & 17280 & 17280 & 17280 & 17280 & 17280 & 17280 & 207360 \\
$\begin{array}{c}\text { Demanda } \\
\text { (m3/mes) }\end{array}$ & 15535 & 14941 & 16054 & 14720 & 10284 & 6938 & 6949 & 7130 & 8937 & 11973 & 12570 & 11780 & 137811 \\
$\begin{array}{c}\text { Déficit/ } \\
\text { exceso }\end{array}$ & 1745 & 2339 & 1226 & 2560 & 6996 & 10342 & 10150 & 8343 & 5307 & 4710 & 5500 & 5500 & 69549 \\
\hline
\end{tabular}


Tabla 4. Balance hídrico proyectado para el Olivo

\begin{tabular}{lcccccccccccccccccccccc}
\hline Descripción & $\mathbf{E}$ & $\mathbf{F}$ & $\mathbf{M}$ & $\mathbf{A}$ & $\mathbf{M}$ & $\mathbf{J}$ & $\mathbf{J}$ & $\mathbf{A}$ & $\mathbf{S}$ & $\mathbf{O}$ & $\mathbf{N}$ & $\mathbf{D}$ & $\begin{array}{c}\text { mm/ } \\
\mathbf{a n u a l}\end{array}$ \\
\hline $\begin{array}{l}\text { Oferta } \\
(\mathrm{m} 3 / \mathrm{mes})\end{array}$ & 17280 & 17280 & 17280 & 17280 & 17280 & 17280 & 17280 & 17280 & 17280 & 17280 & 17280 & 17280 & 207360 \\
$\begin{array}{l}\text { Demanda } \\
\text { (m3/mes) }\end{array}$ & 9321 & 8965 & 9632 & 8832 & 6170 & 4163 & 4169 & 4278 & 5362 & 7184 & 7543 & 7068 & 82687 \\
$\begin{array}{l}\text { Déficit } \\
\text { lexceso }\end{array}$ & 7959 & 8315 & 7648 & 8448 & 11110 & 13117 & 13002 & 11918 & 10096 & 9737 & 10212 & 10212 & 124673 \\
\hline
\end{tabular}

Tabla 5. Parámetros de diseño

\begin{tabular}{lll}
\hline Descripción & Unidad & $\begin{array}{l}\text { Cultivo } \\
\text { Césped -Olivo }\end{array}$ \\
\hline Tiempo máximo de riego & hora & 3,73 \\
ETC máximo & $\mathrm{mm} /$ día & 4,25 \\
Kc máximo & & 1 \\
Etc. máximo & $\mathrm{mm} /$ día & 4,25 \\
Eficiencia & décimas & 0,85 \\
Distancia de laterales & $\mathrm{mm} /$ día & variable \\
Distancia de laterales & $\mathrm{mm} /$ día & variable \\
Caudal del difusor & $\mathrm{m} 3 / \mathrm{hr}$ & 0,6 \\
Distancia entre difusores & $\mathrm{m}$ & variable \\
Demanda máxima /día & $\mathrm{mm} /$ día & 5 \\
Precipitación horaria & $\mathrm{mm} / \mathrm{hr}$ & 44 \\
Lámina bruta & $\mathrm{mm} /$ día & 5 \\
Tiempo de riego/valvular & $\mathrm{min} /$ día & 7 \\
Numero de válvulas & $\mathrm{Turnos}$ & 32 \\
promedio & $1 / \mathrm{s}$ & 6,66 \\
Caudal por válvula & $\mathrm{m}{ }^{2}$ & 835,16 \\
Área promedio/válvula & & \\
\hline Diseno hidráulico. & &
\end{tabular}

Diseño hidráulico.

(ver Tabla 5), donde se tiene un área total de 10,7 ha, subdividida en cuatro sectores (32 válvulas promedio/ sector).

\section{Sectores de riego}

El área total se ha dividido en cuatro sectores (cada una de ellas compuesta por zonas de riego, cada zona tiene una válvula hidráulica para el inicio o cierre del riego).

Sector 1, de 2,10 ha (Av. Paz Soldán y Choquehuanca)

Sector 2, de 2,54 ha (Calle Choquehuanca y Calle Ernesto Plasencia)

Sector 3, de 2,84 ha (Calle Ernesto Plasencia y Calle Conde de la Monclova y Raymundo Morales)

Sector 4, de 3,21 ha (Calle Conde de la Monclova y Raymundo Morales y Calle Carolina Vargas)

Se riega en forma simultánea cada sector; en el sector 3 se encuentra la estación de bombeo con el reservorio de una capacidad de $800 \mathrm{~m}^{3}$. Cada sector cuenta con un número variable de válvulas (zonas de riego) y cada válvula tiene un tiempo de riego promedio de siete minutos/día para una lámina bruta de $5 \mathrm{~mm} /$ día, considerando una velocidad promedio del difusor de $44 \mathrm{~mm} / \mathrm{hr}$.

Zonas de riego (electroválvulas, tubería de PVC, PE y difusores)

Las electroválvulas comandarán un área que corresponda a un caudal máximo de 7,5 lps (caudal máximo), tienen un número de difusores de diferente diámetro de humedecimiento, distanciados de tal forma que se evite mojar los troncos del olivo, pero regando adecuadamente el césped, se establecerán pequeñas redes de tubería para cada zona de riego, área de influencia de cada válvula (ver Fig. 2).

Los difusores serán del tipo emergente "pop-up" tomando en cuenta los factores de seguridad antirrobos, sectoriales y con la posibilidad de reducción del radio de humedecimiento en $20 \%$ de los radios a utilizar 5,5, $4,6,3,7,3,1,2,4$ y 1,8 m, así como difusores de área de mojado rectangular.

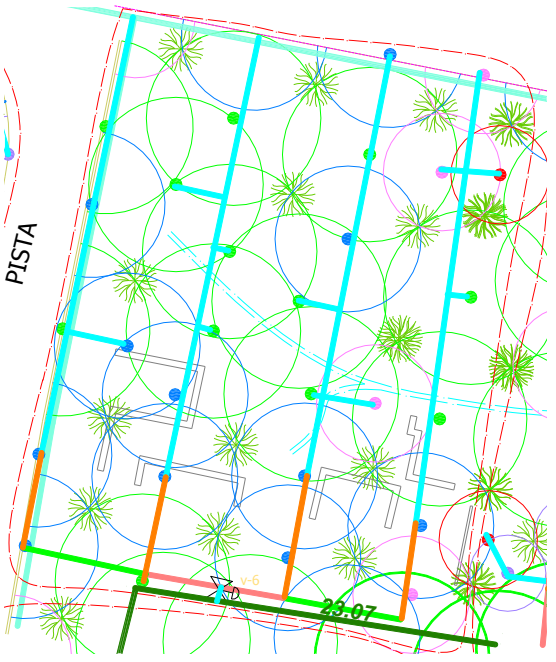

Figura 2. Zona se riego, válvula 6.

\section{Red de riego}

La red estará conformada por tuberías de PVC C-5 U/F, de los siguientes diámetros: $160 \mathrm{~mm}, 110 \mathrm{~mm}, 90 \mathrm{~mm}$, $75 \mathrm{~mm}, 63 \mathrm{~mm}, 11 / 2$ ” y 1 ”. Esta red estará enterrada en zanjas de profundidad variable de $0,4-0,5 \mathrm{~m}$, la tubería tendrá cama de arena en la base y relleno seleccionado encima de la clase del tubo. El diseño de la red de tuberías se empezó por el cálculo de la pérdida de carga en la red matriz para cada uno de los sectores, siguiendo los 
tramos más críticos; posteriormente, se obtuvo la pérdida de carga en las tuberías secundarias por válvula crítica, desnivel topográfico, pérdida en accesorios, lográndose obtener el requerimiento de presión a nivel de cabecera de proyecto, por cada sector. Los análisis de distribución de las pérdidas totales, se ha realizado empleando la fórmula de Hazen Williams para tubería de PVC.

La presión requerida por el sistema sería proporcionada por una batería de bombas en paralelo, porque el sistema requiere presión para superar la fricción en las tuberías de conducción, las pérdidas en el cabezal de riego, en los arcos y porta laterales, además de la presión de trabajo del difusor. Los resultados nos indican que se necesita un carga dinámica total de $50 \mathrm{~m}$, un caudal máximo de $7,5 \mathrm{l} / \mathrm{s}$ por bomba; se utilizará una batería de cuatro bombas, equivalente a $30 \mathrm{l} / \mathrm{s}$ o $108 \mathrm{~m}^{3} / \mathrm{h}$. En el cálculo de la potencia de la bomba, se ha utilizado una eficiencia de $70 \%$ y la eficiencia del motor de $70 \%$, proporcionado una potencia de 8 HP para cada motor.

\section{Capacidad de la cisterna}

La capacidad de la cisterna será de $800 \mathrm{~m}^{3}$, este volumen ha sido obtenido de acuerdo con el siguiente balance semanal y la operación del sistema de riego para el mes de marzo, mes de máxima demanda por parte del césped. Con la finalidad de optimizar el uso del espacio y teniendo en consideración que la dotación de agua del distrito es discontinua (dos veces por semana), se programó el riego para un periodo de seis días, regando cuatro días. Se consideró que los días que le toca el turno de riego en el distrito, la cisterna actuará como fuente de almacenamiento y bombeo simultáneo para cada turno, almacenando solo lo necesario para otorgar el riego de un día. Por tanto, se estima que el volumen necesario a almacenar es de $623 \mathrm{~m}^{3}$ aproximadamente, al cual se adiciona un factor de seguridad de 1,28 para posibles eventualidades, alcanzando un volumen total de cisterna de $800 \mathrm{~m}^{3}$ (ver Tabla 6).

\section{Análisis económico}

La propuesta de cambio de sistema de riego engloba la construcción de cisternas, remodelación de la laguna y el sistema de riego a presión para el Bosque del Olivar, lo cual representa un costo de inversión de S/. 3.537.416,79 nuevos soles y un costo de operación y mantenimiento de S/ 434.722,39 nuevos soles.

\section{Beneficios cualitativos}

- Mejora la calidad del sistema de riego del Bosque El Olivar.

- Reduce significativamente el volumen de agua de riego que actualmente se viene empleando.

- Mejora la calidad del servicio de parques y jardines

Tabla 6. Balance hídrico semanal en el mes crítico, marzo y capacidad de la cisterna

\begin{tabular}{|c|c|c|c|c|c|c|c|c|}
\hline Descripción & Lunes & Martes & Miércoles & Jueves & Viernes & Sábado & Domingo & Vol. $\left(\mathrm{m}^{3}\right)$ \\
\hline \multicolumn{9}{|l|}{ Oferta } \\
\hline Caudal disponible ( $1 / \mathrm{s})$ & 50 & & & 50 & & & & \\
\hline Caudal disponible $\left(\mathrm{m}^{3} / \mathrm{hr}\right)$ & 180 & & & 180 & & & & \\
\hline $\begin{array}{l}\text { Horas de disponibilidad de } \\
\text { agua }\end{array}$ & 12 & & & 12 & & & & \\
\hline Volumen disponible $\left(\mathrm{m}^{3}\right)$ & 2160 & & & 2160 & & & & 4320 \\
\hline \multicolumn{9}{|l|}{ Demanda } \\
\hline Riego todos los días(m³/día) & 534 & 534 & 534 & 534 & 534 & 534 & 534 & 3738 \\
\hline Riego sin domingo ( $\mathrm{m}^{3} /$ día $)$ & 623 & 623 & 623 & 623 & 623 & 623 & 0 & 3738 \\
\hline Turnos de riego & 12 horas & & & 12 horas & & & & \\
\hline $\begin{array}{l}\text { Volúmenes de agua aplicar } \\
\left(\mathrm{m}^{3}\right)\end{array}$ & 124 & & 623 & 1246 & & 623 & & 3738 \\
\hline Días de Riego & $\mathrm{x}$ & & $\mathrm{x}$ & $\mathrm{x}$ & & $\mathrm{x}$ & & \\
\hline $\begin{array}{l}\text { Caudal aplicar en } 12 \text { horas } \\
\left(\mathrm{m}^{3} / \mathrm{hr}\right)\end{array}$ & 104 & & & 104 & & & & \\
\hline Caudal aplicar ( 1/s ) & 29 & & & 29 & & & & \\
\hline $\begin{array}{l}\text { Caudal aplicar en } 6 \text { horas } \\
\left(\mathrm{m}^{3} / \mathrm{hr}\right)\end{array}$ & & & 104 & & & 104 & & \\
\hline $\begin{array}{l}\text { Caudal aplicar en } 6 \text { horas } \\
(1 / s)\end{array}$ & & & 29 & & & 29 & & \\
\hline $\begin{array}{l}\text { Capacidad mínima de } \\
\text { reservorio (m3) }\end{array}$ & & 623 & & & 623 & & & \\
\hline Balance (m3) & & & & & & & & 581 \\
\hline
\end{tabular}

Considerando factor de seguridad de 1.28 el reservorio tiene una capacidad efectiva de $800 \mathrm{~m}^{3}$. 
que se presta a los vecinos.

- Permite disminuir el nivel de elementos vectores de enfermedades, en particular coliformes fecales así como roedores.

- Contribuye a la puesta en valor de esta área.

- Refuerza significativamente la imagen de modernidad y ornato, acordes con el distrito.

- Logra la disipación parcial de la sensación térmica en épocas de verano.

- Mejora la regulación de la humedad ambiental.

- Incrementa la disponibilidad del agua de riego en el distrito, posibilitando atender otros requerimientos.

\section{Beneficios cuantitativos}

Valor presente del bosque

Se tomó la metodología de valoración contingente, a fin de poder estimar la disposición de la población de San Isidro a pagar por la conservación del bosque. Se conoce que la población del distrito es de 58.056 habitantes (censo del INEI 2007) y la población económicamente activa - PEA es de 49.722 habitantes. A partir de esta población referencial (PEA), se estableció el tamaño de muestra de la encuesta de 150 personas.

A fin de estimar los rangos entre los cuales se establecerá un valor referencial del "aporte a pagar" por la población, se tomó como base la escala del arbitrio de parques y jardines establecida por la Municipalidad de San Isidro, la cual se encuentra estratificada en función a la cercanía y disfrute de áreas verdes por lo ocupantes de las edificaciones; de este modo se tiene que mientras un predio que se halla dentro de las áreas verdes paga $\mathrm{S} /$. $1,20 \mathrm{~m}^{2}$, un predio que se encuentra en una avenida $\sin$ mucho acceso a áreas verdes paga $\mathrm{S} / .0,050 \mathrm{~m}^{2}$.

Teniendo en consideración que la percepción de pertenencia de la población a un lugar, al igual que las valoraciones sentimentales, son independientes de las distancias y que la importancia que cada individuo le otorga a su entorno social y físico tiene una estrecha relación con el sentimiento de pertenencia en la psiquis de la comuna, el asumir el mismo criterio empleado en la estratificación de la escala del arbitrio basada en la cercanía a las áreas verdes, generaría información poco representativa, dado que son contextos distintos. No obstante, al ser estas las tasas que actualmente se vienen aplicando con éxito por la institución, cuyo mejor indicador de aceptación y efectividad es la baja tasa de morosidad registrada, se deduce que el rango establecido es aceptado por la población.

Considerando una amplitud del rango de $120 \%$ y tomando como promedio $\mathrm{S} /$. 13,76 de recaudación per cápita por el mantenimiento del Bosque del Olivar, se tradujo las variaciones del rango a moneda nacional. En función a las cifras calculadas, se incluyó en la encuesta la siguiente pregunta:

Se obtuvo que el $65 \%$ de la muestra señaló estar dispuesta a pagar S/. 15 y un $15 \%$ adicional estuvo de acuerdo con realizar una contribución mayor a ello. En una pregunta previa: "Teniendo en cuenta sus ingresos, gastos y preferencias personales, ¿estaría Ud. dispuesto a pagar por mantener la intangibilidad del Bosque el Olivar?" se obtuvo que más del $81 \%$ de la muestra estuvo de acuerdo con realizar alguna contribución para preservar el bosque. Finalmente, ambos resultados nos permiten inferir que en su mayoría la población del distrito estaría dispuesta a pagar S/. 15 a fin de mantener la intangibilidad del bosque el Olivar de San Isidro. En este orden de ideas anualmente el monto aportado por la población sería: 49.722 hab. (PEA) X S/. 15 hab/año = S/. 745.830 anuales.

\section{Valor de la producción del olivo}

En el caso de la situación sin proyecto los paramentos para el cálculo de los beneficios son los siguientes:

El Olivar tiene un extensión de 10,7 ha, considerando que en la actualidad no todo el bosque está cubierto de Olivos, se asume para efectos productivos un área neta de 8,56 ha (reducción del $20 \%$ ).

Área de terreno 8,56 ha, rendimiento $2,5 \mathrm{TN} /$ ha, precio $9,5 \mathrm{~S} / . / \mathrm{kg}$ procesado y empacado.

Ingresos en el año $0,8,56$ ha $\times 2,5 \mathrm{TN} /$ ha $\times 1$ campañas/ año $=21,4$ TN/año

\section{$21.400 \mathrm{~kg} / \mathrm{año} \times \mathrm{S} /$. 9,50/kg. = S/. 203.300/año}

Se prevé que de no darse ninguna intervención el rendimiento disminuirá $1 \%$ anualmente.

\section{Bonos de carbono}

El promedio de generación de toneladas de carbono por hectárea que un bosque podría producir es de 20,14 TN/ha (Ministerio del Medio Ambiente - Consultora ambiental Libélula); adicionalmente, se tiene que el valor internacional por toneladas de dióxido de carbono capturado (bono de carbono) es de US\$ 10. Dadas las condiciones de las áreas verdes del bosque se ha considerado que la capacidad de captura de $\mathrm{CO} 2$ se ve reducida en un $10 \%$ respecto del promedio y que de no darse intervención alguna esta disminuirá $1 \%$ anualmente.

En este sentido, en la situación sin proyecto los paramentos para el cálculo de los beneficios por captura de carbono son los siguientes: Área de terreno 10,7 ha, captura de CO2 de 18,13 (TN/ha), valor del bono (10 US\$ /TN) y tipo de cambio 3,00 (S/./US\$), Ingresos en el año 0: 10,7 ha $\mathrm{x} 18,13 \mathrm{TN} /$ ha X S/.30/ TN = S/. 5.818,45 año.

En el caso de la situación con proyecto, los paramentos para el cálculo de los beneficios son los mismos que en la situación sin proyecto. Sin embargo, se prevé que al mejorar las condiciones del bosque, la capacidad de captura de $\mathrm{CO} 2$ se recuperará en el 5to año y se mantendrá constante a partir del mismo en $20,14 \mathrm{TN} /$ ha.

\section{Análisis de rentabilidad}

El análisis de rentabilidad fue desarrollado comparando el costo de oportunidad del capital a invertir bajo la metodología del costo-beneficio; en esta, el flujo de caja del proyecto está en función de los costos del mismo y los ingresos que potencialmente puedan captarse como son el valor presente de bosque, estimado bajo la metodología de valoración contingente, el valor de la producción del olivo y la valorización de la potencialidad de producción 
de bonos de carbono del bosque. Bajo esta metodología se estimaron indicadores de rentabilidad como el Valor Actual Neto (VAN), la Tasa Interna de Retorno (TIR) y el Ratio Beneficio-Costo (B/C).

Respecto del VAN, el valor calculado es de S/. 1.748.633,04 para una tasa de $11 \%$, el cálculo de la TIR arrojó un resultado de $27 \%$, muy superior a la tasa de rentabilidad social estimada por el estado de $11 \%$, el ratio Beneficio-Costo (B/C) de S/. 1,51.

\section{Conclusiones}

El olivo tiene una demanda hídrica de $657,47 \mathrm{~mm} / \mathrm{año}$, menor que la del césped que es de 1.095,79 mm/año; al cubrir la demanda hídrica del césped se cubre la demanda del olivo y, para efectos de cálculo, el volumen de agua requerido será el que demande el riego del césped en el sistema de riego por aspersión (difusores), utilizando una alta eficiencia de riego y evitando inundaciones que perjudiquen al olivo.

Al comparar la dotación de agua y la aplicación bajo las condiciones actuales, se evidencia que en las 10,7 ha que conforman el Bosque del Olivar, para el caso del césped, existe un déficit anual de agua de $26,919 \mathrm{~m}^{3}$, pero que se hace crítico en los meses de verano. En cambio, bajo el supuesto de que las 10,7 ha del bosque solo están cubiertas por olivos, en función a los cálculos realizados, se ha identificado que existe sobre aplicación de agua de $66,661 \mathrm{~m}^{3}$ acumulados anuales.

El balance hídrico sin proyecto permite conocer la actual situación del sistema de riego, determinándose la existencia de una sobre oferta de agua para el Olivar, lo cual produce encharcamientos e inundaciones en las áreas cercanas a las raíces produciendo pudrición de las mismas.

Con la finalidad de optimizar el uso del espacio y considerando que la dotación de agua del distrito es de dos veces por semana, lunes (12 horas) y jueves (12 horas), la cisterna se operará como reservorio para el bombeo durante el turno de riego y se almacenará solo lo necesario para otorgar el riego de un día de acuerdo a la operación planteada, por lo que se determina la capacidad del almacenamiento de $800 \mathrm{~m}^{3}$.

El cambio del sistema de riego nos permitirá mantener al cultivo del olivo en mejores condiciones agronómicas (evita inundaciones), además de realizar el uso eficiente del recursos hídrico. Se estimó que el cambio de sistema de riego tiene un monto de inversión de S/. 3.537.416,79 nuevos soles y costos de Operación y Mantenimiento anual de S/. 434.722,39.
Se determinó como beneficios acumulados del proyecto, para un horizonte de 10 años, el valor del bosque en S/. 8.204.130,00; el valor de la producción del olivo en S/. 2.236.300,00 y el valor de la captación de bonos de carbono en S/. 69.174,86. En base a estas proyecciones estimadas, se realizó la evaluación de rentabilidad que demostró ser positiva en el tiempo con indicadores como un TIR de $27 \%$ y un Ratio Beneficio-Costo (B/C) de S/. 1,51 siendo viable el proyecto.

\section{Literatura citada}

Castañón, G. 2000. Ingeniería de Riego, Utilización Racional del Agua. ( $1^{\circ}$ edición). Madrid - España.

CEDEX. 1994. Máster en Ingeniería de Regadios Riego Localizado, Tomo IX. Madrid - España: Centro de Estudios y Experimentación de Obras Públicas.

Celiz Guevara, C. 2001. Evaluación de los Sistemas de riego por gravedad y goteo en el cultivo de caña de azúcar en los campos de la Agroindustria Paramonga, ubicados en la cuenca del Rio Fortaleza. Lima - Perú: Universidad Nacional Agraria La Molina.

Organización de las Naciones para la Agricultura y Alimentación (FAO). 2006. Evapotranspiración del Cultivo. Manual 56. (1ra Edición).

Fuentes Yague, J.L. 1998. Técnicas de riego. (3º edición). Madrid - España: Ministerio de Agricultura y Alimentación de España.

García Castillas, I. 1997. Sistemas de riego por aspersión y goteo. ( $1^{\circ}$ edición). México.

Pizarro Cabello, Fernando. (1996). Riegos Localizados de Alta Frecuencia. ( $3^{\circ}$ edición). Madrid - España: Ediciones Mundi - Prensa.

Torres Rúa, A. 2001. Evaluación Técnica y Económica del Sistema de Riego por Goteo en el fundo "Agrocurumuy". Lima - Perú: Universidad Nacional Agraria La Molina.

Pascual, B. 2007. Riego de gravedad y presión. España: Universidad Politécnica de Valencia.

Sifuentes Alegría, K. 2008. Evaluación de un Sistema de Riego por goteo en el Cultivo de Esparrago en el Fundo San Miguel - Virú, (Trujillo - la Libertad). Perú: Universidad Nacional Agraria La Molina.

Vera y Moreno Consultores. 2006. Plan Integral del Bosque el Olivar. 